\title{
SOZIALISATION UND GESCHLECHT - STRITTIGE POSITIONEN
}

Mechthild Bereswill \& Gudrun Ehlert

Universität Kassel

E-Mail: bereswill@uni-kassel.de

URL: https://www.uni-kassel.de/fb01/institute/sozialwesen/fachgebiete/soziologie-sozialer-differenzierung-und-soziokultur/prof-dr-mechthild-bereswill.html

Hochschule Mittweida

E-Mail: ehlert@hs-mittweida.de

URL: https://www.sw.hs-mittweida.de/professuren/prof-dr-phil-gudrun-ehlert.html

Zitationsvorschlag:

Bereswill, Mechthild/Ehlert, Gudrun (2020): Sozialisation und Geschlecht - strittige Positionen. In: Gesellschaft - Individuum - Sozialisation (GISo). Zeitschrift für Sozialisationsforschung 1 (1). DOI: 10.26043/GISo.2020.1.1

Link zum Artikel:

https://doi.org/10.26043/G/So.2020.1.1

(C) 


\title{
SOZIALISATION UND GESCHLECHT - STRITTIGE POSITIONEN
}

\author{
Mechthild Bereswill \& Gudrun Ehlert
}

\begin{abstract}
Ansätze der sozial- und kulturwissenschaftlichen Geschlechterforschung stehen in der Kritik, die „biologische Natur“ des Menschen nicht zu berücksichtigen. Dies gilt insbesondere im Zusammenhang von Fragen der Geschlechtersozialisation und angrenzender pädagogischer Felder. Gegen grundlegende Einsichten der Geschlechterforschung werden deshalb neurowissenschaftliche Forschungsbefunde und evolutionspsychologische Annahmen angeführt und als Nachweis dafür geltend gemacht, dass Geschlechterunterschiede stabil und biologisch begründet seien. Im vorliegenden Beitrag wird zunächst an Perspektiven der sozialwissenschaftlichen Geschlechterforschung angeknüpft. Anschließend werden ausgewählte Befunde und Diskussionen der Neurowissenschaften und Evolutionspsychologie analysiert. Im Ausblick wird am Beispiel von Geschlecht dargelegt, warum eine Integration von biologischer Forschung und sozialwissenschaftlicher Sozialisationsforschung problematisch ist.
\end{abstract}

Keywords: Geschlechtertheorien, Sozialisationsforschung, Neurowissenschaften, Anlage-Umwelt-Debatten

\section{EINLEITUNG}

Eine biologische Natur des Menschen gibt es nur in Form anthropologischer Konstanten - zum Beispiel Weltoffenheit und Bildbarkeit des Instinktapparates. Die anthropologischen Konstanten machen die sozio-kulturellen Schöpfungen des Menschen möglich und beschränken sie zugleich. Die jeweilige Eigenart, in der Menschenhaftigkeit sich ausprägt, wird umgekehrt aber bestimmt durch diese sozio-kulturellen Schöpfungen und gehört zu deren zahlreichen Varianten. So kann man zwar sagen: der Mensch hat eine Natur. Treffender wäre jedoch: der Mensch macht seine eigene Natur - oder noch einfacher: der Mensch produziert sich selbst (Berger/Luckmann 2004: 51f.).

Wir beginnen unsere Überlegungen zum Verhältnis von Sozialisation und Geschlecht mit einem theoretischen Argument, das Peter L. Berger und Thomas Luckmann in den 1960erJahren formuliert haben. Aus ihrer Perspektive ist die Vergesellschaftung des Menschen ein durch spezifische kulturelle Konstellationen strukturierter Prozess der notwendigerweise eigentätigen Hervorbringung von Gesellschaft. Für eine sozialwissenschaftliche Auseinandersetzung mit Sozialisation ist dabei die Feststellung der Wissenssoziologen Berger und Luckmann, eine „biologische Natur des Menschen“ gebe es nicht, hoch aktuell. Denn Behauptungen einer biologischen Determiniertheit menschlichen Handelns genießen gegenwärtig hohes Ansehen, sowohl in wissenschaftlichen als auch in populären Diskursen. Vor diesem Hintergrund ist eine kritische Auseinandersetzung mit Auffassungen, die eine ganz bestimmte biologische Ausstattung des Menschen als Ursache für dessen soziales Handeln voraussetzen, grundlegend für sozialwissenschaftliche Konzeptionen von Sozialisation. So begreifen Berger und Luckmann die Vergesellschaftung des Menschen eben nicht als biologisch vorbestimmt. Sie gehen hingegen davon aus, dass die Angewiesenheit des Menschen auf soziale Kontexte mit den anthropologischen Spezifika seiner „physiologischen Frühgeburt" zusammenhängt (Portmann 1955, zitiert nach Lenz 2011: 386; Berger/Luckmann 2004: 50). Da die Entwicklung des menschlichen Organismus bei der Geburt noch nicht abgeschlossen ist, vollziehe sich der "Vorgang der Menschwerdung" notwendigerweise in „Wechselwirkung mit der Umwelt" (Berger/Luckmann 2004: 51). Mit ihrer Auffassung von der „Umwelt“ des Menschen beziehen sich Berger und Luckmann auf biologische Ansätze zu den Umwelten von Tieren und Menschen (von Uexküll 1956). Demnach ist die „Umweltbeziehung des Menschen“ nicht 
durch eine fest gelegte und begrenzte Umgebung strukturiert, sondern durch dessen spezifische "Weltoffenheit" gekennzeichnet (Berger/Luckmann 2004: 49 f.). Daraus folgt, dass der Mensch sich aufgrund seiner spezifischen Umweltbeziehung aktiv vergesellschaften muss, da seine geringe biologische Festlegung inm eine große Weltoffenheit und Plastizität mitgibt. Der Mensch „produziert“ auf dieser Basis nicht nur „sich selbst“, er bringt im Austausch mit anderen Menschen, auch in der intergenerationalen Relation, Gesellschaft hervor.

Diese Perspektive auf Sozialisationsprozesse steht in scharfem Kontrast zu Auffassungen, die den Menschen als biologisch und evolutionär determiniertes, wenngleich lernfähiges Wesen konzipieren, soziales Verhalten in letzter Konsequenz aus dessen "biologischer Natur" ableiten und von einem "Anlage-Umwelt-Verhältnis" ausgehen. Solche Konzepte werden im deutschsprachigen Raum verstärkt seit der Jahrtausendwende vertreten, wobei sich eine Bandbreite von stark deterministischen bis hin zu bio-psychosozialen Ansätzen findet. Eine Schlüsselrolle übernehmen dabei die Neurowissenschaften im Hinblick auf allgemeine Fragen, was den Menschen ausmacht (Schmitz/Höppner 2014: 2). Entscheidend ist hierbei die mediale Vermittlung neurowissenschaftlicher Befunde als „Fakten“ in die Öffentlichkeit und in die Pädagogik (ebd.: 7).

Auch im Kontext geschlechterpädagogischer Fragen und entwicklungspsychologischer Grundlagen wird zunehmend auf neurowissenschaftliche und evolutionspsychologische Ansätze Bezug genommen (Pauen 2016; Matzner/Tischner 2012; Bischof-Köhler 2011; Steins 2010). In diesem Zusammenhang zeigen sich unmittelbare Schlüsse zwischen naturwissenschaftlichen Forschungsansätzen und Befunden einerseits und dem Verhalten bzw. den Entwicklungspotenzialen von Kindern und Jugendlichen andererseits. So werden beispielsweise neurowissenschaftliche Forschungen zur Wirkung von Androgenen (Steroidhormone wie Testosteron) auf die Hirnentwicklung von Menschen dazu herangezogen, Unterschiede zwischen den Geschlechtern biologisch zu begründen.
Je höher die Konzentration von Androgenen während der Foetalentwicklung, umso stärker war im Grundschulalter die Neigung von Jungen und Mädchen zu jungentypischen Beschäftigungen (Aueyung et al. 2009). Zur Vollständigkeit ist zu erwähnen, dass die Testosteronausschüttung auch bei erwachsenen Männern eine direkte Wechselwirkung mit dem männlichen Wettkampfverhalten aufweist. Androgene intensivieren die Kampfeslust, bei Erfolg steigt die Konzentration, bei Misserfolg fällt sie ab. (Bischof-Köhler 2010: 164)

Aus der Sicht einer sozialwissenschaftlich fundierten Sozialisationsforschung ist diese Argumentation ein typisches Beispiel für das, was Berger und Luckmann in Frage stellen. Behauptet wird eine universale „biologische Natur des Menschen", die dessen Verhalten bis in den Alltag steuert. Diese basiere auf natürlichen Geschlechterunterschieden, die pränatal angelegt seien. So wird eine direkte Verbindung gezogen von der hormonell beeinflussten Entwicklung des Fötus im Mutterleib bis hin zum Kampfverhalten erwachsener Männer in der Gegenwartsgesellschaft.

Für die sozialwissenschaftliche Geschlechterforschung handelt es sich bei solchen Argumentationen um die Naturalisierung sozialer Phänomene, deren Komplexität auf einen Kausalzusammenhang reduziert wird (HagemannWhite 1984; Gildemeister/Hericks 2012). Diese Auffassung teilen Ansätze der naturwissenschaftlichen Geschlechterforschung, die den Geschlechterbias der wissenschaftstheoretischen Grundlagen solcher Biologisierungen aufdecken (Ebeling 2006; Ebeling/Schmitz 2006; Palm 2008, 2012). Die unterkomplexe Argumentation, gesellschaftliche Phänomene naturwissenschaftlich zu begründen, unterläuft zudem generelle sozialwissenschaftliche Auffassungen von Vergesellschaftung. Aber auch in den naturwissenschaftlichen Debatten über das Verhältnis von Hormonen, Hirnfunktionen und Geschlechterdifferenzierung werden solche Zusammenhänge wesentlich zurückhaltender diskutiert, als dies in pädagogischen und populären Diskursen den Anschein hat (Hyde 2005; Hausmann 2007; Hirnstein/Hausmann 2010; Jordan 2010; Siegler et al. 2016; Hustvedt 2018). 
Im Folgenden untersuchen wir diese Debatte über Sozialisation und Geschlecht. Im nächsten Schritt skizzieren wir wesentliche Argumentationslinien der sozialwissenschaftlichen Geschlechterforschung zu Sozialisation (2.). Anschließend kontrastieren wir einen Ausschnitt aus den bereits erwähnten naturwissenschaftlichen Ansätzen, die von einer natürlichen, binär strukturierten Geschlechterdifferenz ausgehen und fragen nach den Konsequenzen solcher Setzungen (3.). Im Ausblick plädieren wir für eine Weiterentwicklung und Stärkung der sozialwissenschaftlichen Geschlechterforschung zu Sozialisation (4.).

\section{GESCHLECHTERSOZIALISATION ALS KOMPLEXER PROZESS}

Gesellschafts- und kulturwissenschaftliche Ansätze zur Herausbildung von Geschlechterverhältnissen, Geschlechterordnungen und Geschlechteridentitäten beziehen sich auf unterschiedliche Theorietraditionen und Forschungsansätze, die kein einheitliches Verständnis der Kategorie Geschlecht erlauben. Es handelt es sich um eine vielstimmige und kontroverse Theorie- und Forschungslandschaft. Die besondere Qualität dieser inter- und transdisziplinären Diskurse liegt in der fortwährenden Reflexion der eigenen Konzepte und Forschungsansätze (Bereswill 2008; Althoff et al. 2017; Kortendiek et al. 2019). Dennoch werden zentrale Konzepte und Erkenntnisse der Geschlechterforschung und Queer Studies unter Bezug auf evolutionspsychologische und neurowissenschaftliche Forschungsbefunde immer wieder in Frage gestellt, wobei das Konzept von "Geschlecht als soziale Konstruktion" im Fokus steht. In Abgrenzung dazu verallgemeinern die Kritiker*innen ein naturwissenschaftliches Paradigma zur Erklärung von Geschlechterunterschieden, und einzelne gehen soweit, Ansätze der sozial - und kulturwissenschaftlichen Geschlechterforschung als unwissenschaftliche Ideologie abzuwerten (Matzner/Tischner 2008: 12 f.; Bischof-Köhler 2010: 154 f.; Krebs 2012: 23 f.; Strüber 2012: 46 f.).

Über diese Entwicklung schreibt die US-amerikanische Literaturwissenschaftlerin und Schriftstellerin Siri Hustvedt in ihrem Essayband "Die Illusion der Gewissheit", sie sei die „selbstgefälligen Annahmen über hormonell oder psychologisch bedingte Geschlechterunterschiede leid“ (2018: 44). Sie unterstreicht, „dass starre Konzepte von Biologie und Natur in Verbindung mit bestimmten Spielarten der Evolutionstheorie seit langer Zeit vermeintliche Wahrheiten über psychologische Geschlechtsunterschiede befördern" (ebd.: 148 f.). Hustvedts genervte Bewertung des Diskurses als „selbstgefällig" verdeutlicht, dass es sich nicht um nüchterne wissenschaftliche Kontroversen, sondern um (wissenschafts-)politische Debatten handelt, in denen unvereinbare Wissenskulturen und Forschungsparadigmen aufeinanderprallen. So bezeichnet der Erziehungswissenschaftler und Biologe Michael Lenz die kritische Abgrenzung sozialwissenschaftlicher Positionen gegenüber biologischen Ansätzen als „Biophobie“ (1999: 159) und legt damit die Assoziation nahe, die Kritik sei neurotisch, also unwissenschaftlich.

Tatsächlich wird das Verhältnis von Biologie und Geschlecht in der naturwissenschaftlichen Geschlechterforschung differenziert untersucht ( $E$ beling/Schmitz 2006). So betont Kerstin Palm wissenschaftstheoretische und erkenntniskritische Analysen zum Geschlechterbias der Biologie und hebt die Kontextualisierung und Historisierung des Faches durch sozial- und kulturwissenschaftliche Studien hervor (Palm 2008, 2012). Gleichzeitig unterstreicht sie, dass feministische Biolog*innen Methoden und Konzepte innerhalb des naturwissenschaftlichen Paradigmas zu verändern suchen. Dabei weist sie darauf hin, dass beide Stränge nicht leicht zu verbinden sind. „Wie sich eine sozial- und kulturwissenschaftliche Reflexion, d.h. eine elaborierte theoretische Metaebene, mit einer biologischen Intervention, d.h. einer handlungsorientierten empirischen Forschung ertragreich verbinden könnte, ist bis heute eine noch weitgehend ungelöste Frage“ (Palm 2008: 851, Herv. i. Orig.).

Sozial- und kulturwissenschaftliche Ansätze zu Sozialisation und Geschlecht diskutieren das Verhältnis von Biologie und Geschlecht ebenfalls unterschiedlich. Gleichwohl zeigt sich die übergreifende Auffassung, dass Naturalisierungen und Ontologisierungen von Geschlechterunterschieden keinen angemessenen Zugang zu Fragen der Geschlechtersozialisation erlauben. Theoretisch und methodisch bedeutet dies, dass 
Sozialisationsforschung keine Unterschiede voraussetzt, sondern deren Hervorbringung im Prozess der Vergesellschaftung rekonstruiert. Geschlechterunterschiede sind demnach Ausdruck der von Berger und Luckmann konstatierten "sozio-kulturellen Schöpfungen“ (2004: 51) des Menschen in dem Sinne, dass es sich um kollektive und individuelle Deutungen von verkörperter Differenz handelt, die in den modernen Gesellschaften immer noch weitgehend binär strukturiert ist (weiblich-männlich). Mit anderen Worten: Verhaltensweisen, Persönlichkeitseigenschaften und Identitätsentwürfe von Menschen sind nicht das Resultat einer ganz bestimmten biologischen Ausstattung, sondern Ausdruck ihrer fortlaufenden Vergesellschaftung, die auch Vergeschlechtlichung bedeutet (Gildemeister/Hericks 2012: 261 ff.; Knapp 2014: 61 ff.; Becker-Schmidt 2017: 51 ff.). Sozialisationsprozesse weisen aus dieser Perspektive über die subjektive Entwicklung des Individuums hinaus auf ungleich verteilte gesellschaftliche Chancenstrukturen, auch zwischen den Geschlechtern. Solche Ungleichheitsverhältnisse können aus soziologischer Perspektive nicht naturwissenschaftlich erklärt werden.

Gerade weil Geschlecht immer wieder als ,natürliche' Grundlage menschlichen Lebens gedeutet wurde und wird, ist es umso wichtiger, sich klarzumachen, dass sich Auffassungen zum Verhältnis von Natur und Gesellschaft im Laufe der Jahrhunderte grundlegend geändert haben. Eine Geschlechtersoziologie kommt daher nicht umhin, das Verhältnis natürlicher Grundlagen des menschlichen Lebens zur gesellschaftlichen Herstellung und Deutung von Natur zu reflektieren. Der Gedanke, dass Natur nur als gesellschaftlich gedeutete für diese Aufgabe herangezogen werden kann, ist nicht nur dem Alltagsdenken vergleichsweise fremd. (Gildemeister/Hericks 2012: 309 f., Herv. i. Orig.)

Der im wissenschaftlichen wie populären Diskurs "fremde“ Gedanke, dass Natur als gedeutete immer schon Kultur ist, ist wegweisend für aktuelle Ansätze der Geschlechterforschung, wobei die Frage, "was Biologie ist und bedeutet" (Hustvedt 2018: 178), auch in diesem Feld keineswegs abschließend beantwortet werden kann. Entscheidender ist an dieser Stelle die Betonung einer Perspektive, die naturwissenschaftliche Setzungen als gesellschaftlich kontextgebundene Phänomene reflektiert: „Die Frage bleibt, wann und wie aus Kultur Biologie wird. Radikal zugespitzt lautet sie: Inwiefern können biologische Tatsachen kulturell bedingt sein? Oder vielleicht besser: Wie verkörpern sich Vorstellungen?" (ebd.: 137).

Indem Hustvedt Biologie nicht als vorgängig aus dem Gesellschaftlichen ausklammert und den stofflichen Körper als mental geformtes Wahrnehmungsorgan reflektiert, lenkt sie den Blick auf die Kontextgebundenheit biologischer Erscheinungen. Deren Realität (,,Tatsachen“) leugnet sie nicht, sie setzt sie aber auch nicht als eine unabhängige Größe, die Soziales oder Kulturelles universal steuert. Dabei vollzieht sie einen argumentativen Perspektivwechsel auf das Verhältnis von Biologie und Kultur. Biologie - genauer: biologisches Wissen - alltägliches wie wissenschaftliches -, auch über eigene und fremde Körper und deren Materialität - wird aus diesem Blickwinkel durch kulturelles Wissen und menschliche Erfahrungen und auch durch Emotionen mit hervorgebracht. Diese Denkfigur räumt weder der Biologie noch der Kultur eine Definitionshoheit ein.

Die Perspektive, „biologische Tatsachen“ seien kulturell hervorgebracht, korrespondiert mit dem soziologischen Argument, dass Auffassungen von Natur immer schon gesellschaftlich gedeutet seien, und passt zur Komplexität von Sozialisationstheorien, die Prozesse der Vergesellschaftung als wechselseitige Konstitution von Subjektivität und Gesellschaft reflektieren (Berger/Luckmann 2004; Hurrelmann 1983; Hagemann-White 1984; Bilden 1991; Maihofer 2002; Bilden/Dausien 2006; Rieker 2013). Fragestellungen zur Geschlechtersozialisation richten sich entsprechend auf die subjektive Aneignung und Verarbeitung von kulturellen Konstruktionen der Geschlechterdifferenz, die nicht einfach übernommen oder zurückgewiesen, sondern eigensinnig gestaltet werden (Maihofer 2002; Lenz 2011; Bereswill/Ehlert 2015).

Erste Arbeiten zu Sozialisation und Geschlecht zielten allerdings auf den Nachweis einer geschlechtsspezifischen Sozialisation, verbunden mit der fraglosen Annahme, Mädchen und Jungen seien aufgrund ihrer differenten 
Sozialisationserfahrungen unterschiedlich. Differenz wurde nicht in der Biologie, sondern in der Gesellschaft verortet. Entsprechend schreibt Ursula Scheu in ihrem 1977 erschienenen Buch „Wir werden nicht als Mädchen geboren - wir werden dazu gemacht":

Die für ursprünglich gehaltenen weiblichen Eigenschaften wie Mütterlichkeit, Emotionalität, soziales Interesse und Passivität sind nicht etwa natürlich weiblich und angeboren, sondern kulturell anerzogen. Wie das durch direkte und indirekte Einflüsse in den ersten Lebenstagen, monaten und -jahren geschieht, kann heute mit wissenschaftlicher Präzision in jeder Stufe der Entwicklung aufgezeigt werden. (ebd.: 7)

Scheus Auffassung von einer geschlechtsspezifischen Sozialisation, die aus ihrer Sicht mit einer fundamentalen gesellschaftlichen Benachteiligung von Mädchen und Frauen verbunden ist, ist aus heutiger Sicht ein klassisches Beispiel für Unterschiedsforschung. So werden Unterschiede zwischen Mädchen und Jungen sowie zwischen Frauen und Männern und ihre ungleich verteilten gesellschaftlichen Chancen zwar als Resultat von Sozialisationsprozessen kritisiert, nicht aber grundsätzlich in Frage gestellt, sondern als gegeben vorausgesetzt. In der deutschsprachigen Debatte wird dies bereits Anfang der 1980er-Jahre durch Arbeiten von Carol Hagemann-White (1984) kritisiert, die sich dabei auch auf internationale Studien stützt, die schon Anfang der 1970er-Jahre keine nennenswerten Verhaltens- oder Persönlichkeitsunterschiede zwischen den Geschlechtern feststellen konnten (Metz-Göckel 2000: 105). Hagemann-White würdigt Scheus Arbeit als „viel versprechende Strategie, dem Biologismus entgegenzutreten“" (1984: 76) und stellt ihren Ansatz zugleich radikal in Frage, weil sie der "Logik des Gegners" folgen würde: „Um mit Belegen aus der positivistischen Empirie die frühe Beeinflussung zu verdeutlichen, gerät Scheu in den Zwang, eine zu große Einheitlichkeit des Verhaltens von Müttern und Vätern, sowie eine gradlinige Wirksamkeit der Erziehungsmaßnahmen zu unterstellen“" (ebd.).

Hagemann-Whites scharf formulierte paradigmatische Kritik an den von Scheu herangezogenen Befunden korrespondiert mit der für die
Geschlechterforschung prägenden Auffassung, weder eindeutige Unterschiede zwischen den Geschlechtern zu unterstellen, noch davon auszugehen, diese Unterschiede gelangten über einen spezifischen Erziehungs- oder Interaktionsstil unmittelbar "in das Kind“. Diese Differenzierung ist bis heute von großer Bedeutung, um unterkomplexe Vorstellungen der Übernahme von Rollen oder der Identifikation mit gleichgeschlechtlichen Vorbildern zu vermeiden. In dieser Perspektive ist Geschlecht eben keine eindeutige oder einheitliche Identitätsdimension, die auf fixen Ausprägungen von Differenz beruht. Geschlecht wird vielmehr als eine soziale Konstruktion aufgefasst und Klassifikationen von Geschlechterdifferenz werden als ordnungsbildend für moderne Gesellschaften verstanden. Diese Zurückweisung von eindeutigen Geschlechterunterschieden bei gleichzeitiger Kritik an gesellschaftlichen Ungleichheitsverhältnissen aufgrund von Geschlecht ist bis heute wegweisend für theoretische und methodische Ansätze der Sozialisationsforschung zu Geschlecht.

Die mittlerweile historische Debatte, auf die wir hier zurückgreifen, weist deutliche Verbindungen zu aktuellen Kontroversen über vermeintlich natürliche Geschlechterunterschiede auf. Die Befunde, die für deren Beleg ins Feld geführt werden, stammen immer noch aus den von $\mathrm{Ha}$ gemann-White kritisierten Forschungstraditionen. Geschlechtersozialisation ist aus ihrer Perspektive im Gegensatz dazu ein kollektiver und individueller Prozess, in dessen Zentrum die kulturelle und symbolische Reproduktion von Zweigeschlechtlichkeit steht. „Die Zweigeschlechtlichkeit ist in jeder Gesellschaft ein System, das mit den ökonomischen und politischen Verhältnissen zutiefst verwoben, aber keineswegs identisch ist" (ebd.: 77). Dies verdeutlicht die Komplexität einer gesellschaftswissenschaftlich fundierten Perspektive auf Geschlechtersozialisation. Zweigeschlechtlichkeit wird als Strukturierungsprinzip der modernen Gesellschaft und als symbolisches Sinnsystem begriffen. Mit anderen Worten: Geschlecht strukturiert Gesellschaft und Sozialisation ist demnach immer vergeschlechtlicht (Gildemeister/Hericks 2012: 261 ff.). Während Scheu Sozialisation als einen stringenten Prozess der gesellschaftlichen Zurichtung durch Erziehung mit dem Resultat eindeutiger Unterschiede thematisiert, 
entwickelt Hagemann-White ein komplexes Theorie- und Forschungsprogramm, mit dem sie verschiedene Theorietraditionen neu ausrichten und verknüpfen will. Entscheidend ist aus ihrer Sicht, dass die von ihr aufgenommenen Perspektiven (Psychoanalyse, Ethnomethodologie, symbolische Anthropologie, neuere marxistische Ansätze der kulturellen Reproduktion) „zwischen Denken und Fühlen, zwischen psychosexueller Entwicklung und kognitiven Leistungen nicht trennen (müssen); und daß sie die Eigengesetzlichkeit von Kultur bzw. von bewußten und unbewußten Vorgängen als symbolisches System in den Blick nehmen“ (1984: 78).

Die Einladung, „aus der bisherigen Theoriegeschichte zu plündern“ (ebd.), beinhaltet eine anspruchsvolle theoretische Programmatik. In deren Zentrum steht die Verknüpfung von subjekttheoretischen, kulturwissenschaftlichen und gesellschaftstheoretischen Prämissen, die sich nicht zu einem einheitlichen Theoriemodell von Sozialisation verdichten. Das ist auch nicht möglich, denn Sozialisation ist ein unabgeschlossener, widersprüchlicher und dialektischer Prozess der Reproduktion und Transformation subjektiver und kultureller Verarbeitungen von Differenz und Hierarchie. Geschlecht ist demnach keine persönliche Eigenschaft, sondern ein gesellschaftlicher Strukturgeber, eine Ordnungskategorie und eine konflikthafte Identitätskategorie (Ehlert 2012; Bereswill 2014, 2018; Bereswill/Ehlert 2015, 2018). Deshalb ist der theoretische Eklektizismus, den Hagemann-White vorschlägt, bis heute inspirierend.

Die theoretischen Debatten der Geschlechterforschung reflektieren fortlaufend die angemessene Erfassung von Geschlecht als eine komplexe und relationale Kategorie, wobei Naturalisierungen und Ontologisierungen von Geschlechterdifferenz als wirkmächtige gesellschaftliche Konstruktionen und diskursive Effekte kritisiert werden. Die Abgrenzung von Ansätzen einer Unterschiedsforschung ging mit der Stärkung von sozialkonstruktivistischen und dekonstruktivistischen Ansätzen einher (Butler 1991; Gildemeister/Wetterer 1992; Maihofer 2002; Dausien 2006; Tervooren 2007; Bührmann et al. 2014). In dieser Perspektive wird die Aneignung von kohärenten Geschlechteridentitäten grundsätzlich in Frage gestellt.
Hierauf richtet sich die eingangs angesprochene grundsätzliche Kritik aus pädagogischer und psychologischer Perspektive. Um sozial- und kulturwissenschaftliche Ansätze der Geschlechterforschung zu widerlegen, werden jedoch Ansätze und Befunde herangezogen, die Geschlecht als Variable einsetzen oder als natürliche Unterscheidung von männlichen und weiblichen Körpern auffassen und ausgehend von dieser Differenz Aussagen über die Unterschiede und Gemeinsamkeiten von Frauen und Männern treffen.

\section{GESCHLECHT - EIN NATÜRLICHER UN- TERSCHIED?}

Naturwissenschaftliche Forschungsansätze, die sich auf Geschlechterunterschiede beziehen, fragen nicht nach dem Zusammenhang von Gesellschaft und Geschlecht und thematisieren gesellschaftliche Einflüsse, wenn überhaupt, als „Umwelt“. Es handelt sich also keinesfalls um Sozialisationsforschung. Wir könnten also feststellen, dass die in die Debatte eingeschleusten Befunde zu "natürlichen“ Geschlechterunterschieden für gesellschaftswissenschaftliche Fragestellungen keine Relevanz haben und sie einfach zurückweisen. Es zeigt sich aber eine überaus wirkmächtige, hegemoniale und ausgesprochen robuste Diskursfiguration, die genauer zu untersuchen notwendig ist. Auffallend ist auch, dass häufig eine Hegemonie der Sozialwissenschaften gegenüber den Naturwissenschaften beschworen wird, die überwunden werden soll. Besonders markant verdeutlicht das die folgende Passage aus dem Geleitwort des Sammelbands Gehirn und Geschlecht (Lautenbacher et al. 2007):

Vor nicht allzu langer Zeit war die Beschäftigung mit Geschlechtsunterschieden in der Psychiatrie, Neurologie und Psychologie weitestgehend verpönt. [...] Geschlechtsunterschiede durften nämlich nur Resultate der Sozialisation sein und galten daher größtenteils als rein historisch-gesellschaftliche Produkte. Der biologischen Psychiatrie, zu deren Vertreter (sic!) ich mich zählen darf, waren solche Unterschiede daher lange Zeit nicht substantiell genug, weil sich diese Disziplin mit den stabilen, biologisch engrammierten Determinanten des Normalen und gestörten Verhaltens beschäftigt. Hier hat sich ein 
dramatischer Perspektivenwechsel in jüngster Vergangenheit ergeben. Es ist mittlerweile fast selbstverständlich anzunehmen, dass genetische (und nicht nur Gene auf dem Geschlechtschromosom) und frühe hormonelle Einflüsse die Struktur und Funktion weiblicher und männlicher Gehirne bedingen. (Holsboer 2007: V)

Hier wird eine paradigmatische Wende behauptet und mit Nachdruck begrüßt. Die eigene Wissenskultur habe Geschlecht deshalb nicht berücksichtigt, weil eine ausschließlich sozialisationstheoretische Herleitung nicht zu den naturwissenschaftlichen Standards des eigenen Fachs passe. Zudem wird hier eine Dominanz der Sozialwissenschaften unterstellt und zugleich sozialwissenschaftlichen Theorien jede Erklärungskraft für die eigenen Fragestellungen abgesprochen. Aus Sicht des Autors ist dies nun vorbei und der direkte Zusammenhang zwischen Hormonen und einer stabilen, determinierten zweigeschlechtlichen Struktur und Funktion des Gehirns gilt als „selbstverständlich“. Diese Argumentationsfigur einer angeblich hegemonialen Sozialwissenschaft und Geschlechterforschung, deren Konjunktur sich ihrem Ende zuneigt, findet sich auch in Debatten über Jungenpädagogik (Matzner/Tischner 2008, 2012).

Ein Blick auf die Forschungsbefunde, auf die sich die Argumentation für die Deutungshoheit naturwissenschaftlicher Erklärungsansätze stützt, fördert allerdings keine konsistente Konzeption von Geschlechterdifferenz zu Tage. In vielen Fällen handelt es sich auch nicht genuin um Geschlechterforschung. Gleichwohl setzen alle im Folgenden herangezogenen Ansätze und Befunde fraglos voraus, dass biologische Zweigeschlechtlichkeit die dominante Form der menschlichen Existenz darstellt und Geschlechteridentitäten dieser binären Logik folgen.

Beispielhaft verdeutlicht dies ein Artikel aus dem Handbuch Gehirn und Geschlecht (Lautenbacher et al. 2007) mit dem Titel „Das transsexuelle Gehirn“ (Cohen-Kettenis et al. 2007). Transsexualität wird von den Autor*innen - in Orientierung an der medizinischen Forschung und Diagnostik als Abweichung von einer "normalen Geschlechtsentwicklung" verstanden. Diese „Abweichung" soll genutzt werden, um den
Zusammenhang von Hormonen und Gehirnfunktionen am Beispiel fortlaufender Hormonbehandlungen zu ergründen. Es handelt sich um eine endokrinologische Fragestellung an den Schnittstellen von Medizin, Psychologie und Biologie mit Bezug zur neurowissenschaftlichen Forschung. Bemerkenswert ist, dass die Autor*innen zum Zusammenhang von Hormonbehandlungen und der Struktur und Funktion des Gehirns keine belastbaren Forschungsergebnisse finden konnten, trotzdem aber von einem "transsexuellen Gehirn" sprechen, das die zukünftige Forschung belegen sollte (ebd.: 138). Das Gehirn wird mit dieser sprachlichen Klassifizierung in mehrfacher Hinsicht "vergeschlechtlicht". Vorausgesetzt wird eine "normale“ geschlechterdifferente Struktur (Hirnareale), verbunden mit differenten Funktionen (z.B. Kognitionen oder Emotionen). Daraus leiten sich dann binäre Zuschreibungen ab, weiblich - männlich, die durch eine als Abweichung konzipierte Transsexualität gefestigt werden. Geschlechtsidentität wird demnach medizinisch definiert - als normal oder als gestört - und diese Parameter korrespondieren mit heterosexueller Weiblichkeit und Männlichkeit. Dies deckt sich mit dem Untertitel des bereits genannten Handbuchs, der lautet: "Neurowissenschaft des kleinen Unterschieds zwischen Frau und Mann" (Lautenbacher et al. 2007).

Den „kleinen Unterschied“ setzt auch die Forschung zu kognitiven Geschlechtsunterschieden voraus, indem sie vergleichend nach den kognitiven Fähigkeiten von Jungen und Mädchen sowie von Frauen und Männern fragt (Hirnstein/Hausmann 2010; Jordan 2010). Ein Befund, der immer wieder referiert wird, bezieht sich auf die visuell-räumlichen Verarbeitungskapazitäten von Menschen. So konstatieren Hirnstein und Hausmann, dass sich auf der Grundlage des gegenwärtigen Forschungsstandes Frauen und Männer in „spezifischen raumkognitiven Aufgaben“ (2010: 81) unterscheiden. Herangezogen werden hierfür Befunde zur "mentalen Rotation“, bei der die größten Unterschiede zwischen Frauen und Männern festgestellt würden. Untersucht wird die Fähigkeit, sich zwei- und dreidimensionale Objekte vorstellen, drehen und vergleichen zu können. In einem mentalen Rotationstest (MRT) werden die Versuchspersonen gebeten, innerhalb von drei Minuten 
Würfelfiguren mental zu rotieren. Bei diesen Tests schneiden Männer generell besser ab als Frauen - so der zunächst verallgemeinerte und viel zitierte Befund (kritisch dazu: Hyde 2005; Schmitz/Höppner 2014: 5; Hustvedt 2018: 143 ff.; Palm 2020: 165). Hirnstein und Hausmann beziehen allerdings weitere Untersuchungen zu diesem Test mit ein, die die Eindeutigkeit von Geschlechterunterschieden deutlich relativieren. Demnach ist die "Stärke des Geschlechtereffekts" (2010: 72) von der konkreten Ausführung des Tests abhängig. Dies betrifft die Zeitbegrenzung des Tests und die kognitiven Strategien, die von den Testpersonen eingesetzt werden, um die Aufgabe zu lösen. Frauen, so der Befund, setzen aufwendigere und fehleranfälligere Strategien ein als Männer (ebd.: 78). Entscheidend ist zudem das Maß an Selbstvertrauen der Testpersonen. So verändern sich die Befunde, wenn vor dem Test bestärkende Einschätzungen vermittelt wurden: „Frauen erzielen höhere Punktzahlen in einem mentalen Rotationstest, wenn sie mit einem positiven Stereotyp [...] konfrontiert werden [...]" (ebd.: 79). Hierzu schreibt Hustvedt:

Außerdem ist es empirisch belegt, dass Frauen bei dreidimensionalen Rotationstests schlechter abschneiden, wenn ihnen zuvor gesagt wird, dass Männer diese Aufgabe besser bewältigen. Dieser Leistungsabfall bei Frauen spiegelt eine 'Bedrohung durch Stereotype' wieder. Der Begriff aus der Rassismusforschung sollte ursprünglich die Auswirkungen von Rassenvorurteilen erfassen, wurde aber bald auf Geschlechterstudien ausgeweitet. (2018: 153)

Die Erweiterung des kognitionspsychologischen Tests durch das sozialpsychologische Konzept „stereotype threat" verändert also die Ergebnisse des Testes (Schmitz/Höppner 2014: 5; Palm 2020: 165). Das heißt, die Geschlechterunterschiede, die der Test belegen soll, unterliegen sozialen Einflüssen. Mit Bezug zu den Überlegungen von Hagemann-White kann festgestellt werden, dass der Einbezug „sozialer Faktoren" (Hirnstein/Hausmann 2010: 79) in die Testanordnung „die Eigengesetzlichkeit von Kultur bzw. von bewußten und unbewußten Vorgängen als symbolisches System" (HagemannWhite 1984: 78) ins Spiel bringt. Damit zeigt sich auch die Grenze von Testverfahren als
Beweisführung. Hirnstein und Hausmann problematisieren allerdings nur die fehlende

Übertragbarkeit von psychometrischen Tests auf den Alltag. Die hier berichteten Geschlechtsunterschiede treten in sehr spezifischen kognitiven Leistungstests auf, deren Alltagsrelevanz schon alleine auf Grund ihrer experimentellen Natur limitiert ist. Trotzdem gibt es scheinbar immer wieder Personen oder Gruppen, die mehr als fragliche Rückschlüsse aus diesen Tests für das Alltagsleben ziehen. (2010: 81 f.)

Konkret bedeutet das, dass beispielsweise Behauptungen zum unmittelbaren Zusammenhang von Mathematikfähigkeit und der Fähigkeit zur mentalen Rotation von Mädchen und Jungen die Evidenz von Forschungsbefunden überdehnen. Allgemein weisen die Autor*innen zudem auf die Spezifik ihrer Methoden hin, die einen unmittelbaren Transfer ihrer Ergebnisse beispielsweise in die pädagogische Praxis nicht erlauben. Zudem relativieren Hirnstein und Hausmann die Belastbarkeit von Befunden einer eindeutigen Geschlechterdifferenz.

Dies unterstreicht auch Kirsten Jordan in einem Überblicksartikel zu Befunden der kognitiven Neurowissenschaften. Sie stellt fest, dass die Gemeinsamkeiten zwischen Frauen und Männern im Verhältnis zu den Unterschieden überwiegen und die Befunde zu „Differenzen sowohl auf der hirnstrukturellen als auch der funktionellen Ebene noch sehr uneinheitlich sind" (2010: 95). Sigrid Schmitz und Grit Höppner betonen für die neurowissenschaftliche Publikationslandschaft ebenfalls, dass es eine Reihe von Beiträgen gibt, die in Meta-Analysen zeigen, wie widersprüchlich Befunde zu vermeintlichen Geschlechterunterschieden sind. Zugleich stellen sie fest, dass Publikationen, die die Unterschiede hervorheben, nach wie vor überwiegen (2014: 3).

Für Jordan sind widersprüchliche Befunde, ähnlich wie für Hirnstein und Hausmann, eine Folge von "methodischen Problemen", aber auch dadurch bedingt, „dass Geschlechtsunterschiede in kognitiven und emotionalen Bereichen verschiedensten Einflussfaktoren unterliegen. Neben den biologischen Faktoren wie den Sexualhormonen spielen hierbei die sozialen und 
Umweltfaktoren eine nicht zu unterschätzende Rolle“ (2010: 95).

Was sagen uns die zitierten Stimmen zu Sozialisation und Geschlecht? Zunächst ist erneut zu bedenken, dass es sich nicht um Sozialisationsforschung handelt und die Wissenschaftler*innen in diesem Feld dies auch nicht für sich in Anspruch nehmen. Dennoch werden die Befunde aus der Kognitionsforschung immer wieder herangezogen, um Fragestellungen zu bearbeiten, die mit Sozialisation zusammenhängen. Zu nennen sind die Medienforschung (Trepte/Reinecke 2010: 239 ff.), Untersuchungen zum Spielverhalten von Kindern oder zur Sozialisation von Mädchen und Jungen in öffentlichen Bildungsinstitutionen (Williams/Pleil 2008; Kasten 2012: 78; Bischof-Köhler 2011: 78 ff.; Siegler et al. 2016: 599). Hinzu kommen Aussagen zu unterschiedlichem Sozialverhalten, zur gesellschaftlichen Arbeitsteilung und zu Fähigkeiten und Talenten, die mit der Berufswahl und dem beruflichen Handeln von Frauen und Männern korrespondieren (Lutchmaya/BaronCohen 2002; Baron-Cohen 2004; kritisch dazu: Hyde 2005; Hustvedt 2018: 143 ff.).

Die Diskrepanz zwischen den komplexen theoretischen und methodischen Anforderungen an Sozialisationsforschung, wie wir sie weiter oben ausgeführt haben, und der Generalisierung von speziellen Forschungsbefunden ist bereits deutlich geworden.

Diese Diskrepanz spitzt sich weiter zu, wenn evolutionspsychologische Schlussfolgerungen mit aktuellen gesellschaftlichen Ungleichheitsverhältnissen kurzgeschlossen werden. Das Ergebnis sind weitreichende Feststellungen über unterschiedliche Eigenschaften, Vorlieben, Fähigkeiten und Präferenzen von Mädchen und Jungen sowie von Frauen und Männern. Damit verbunden sind Zuschreibungen von differenten Verhaltensdispositionen wie Wettbewerbsfähigkeit, Kampfbereitschaft oder Fürsorgeverhalten, die unmittelbar aus der Frühgeschichte der Menschheit abgeleitet werden (Matzner/Tischner 2008: 12 f.; Bischof-Köhler 2012: 60; Krebs 2012: 24 ff.). So lautet ein Buchtitel des Mediziners und Psychologen Simon Baron-Cohen Vom ersten Tag an anders (2004) und die Psychologin Doris Bischof-Köhler vertritt die evolutionspsychologische These, Frauen und Männer seien von „Natur aus anders“ (2011), weil sie für die Arterhaltung sehr unterschiedliche Strategien und Verhaltensweisen der „parentalen Investition“ entwickelt hätten. „Unter parentaler Investition versteht man den Aufwand an Zeit, Energie und Risiko, den ein Elternteil pro einzelnem Kind auf Kosten weiterer potenzieller Nachkommen investieren muss" (Bischof-Köhler 2012: 56). Männer bräuchten im Gegensatz zu Frauen hierbei keine Fürsorgepflichten im engeren Sinne zu erfüllen. Sie müssten nur eine Partnerin finden, die bereit sei, sich mit ihnen gemeinsam fortzupflanzen. Um diese Partnerin müssten die Männer allerdings konkurrieren, die Frauen würden erwählt. Aus diesem „Spannungsverhältnis" resultieren laut Evolutionspsychologie unterschiedliche Dispositionen, die die Verhaltensweisen der Geschlechter bis in die Gegenwartsgesellschaft bestimmen (ebd.: 57 ff.).

Im Zusammenhang mit evolutionären Erklärungen ist zudem immer wieder die Rede davon, „dass Männer mehr als Frauen das Haus verließen, um Jagen zu gehen“" (Haider/Malberg 2010: 117). Diese augenscheinliche Rückprojektion der räumlichen Strukturen von modernen bürgerlichen Gesellschaften auf die Frühgeschichte ist nicht einfach eine sprachliche Ungenauigkeit. Sie verweist vielmehr darauf, dass theoretische Thesen und Forschungsbefunde immer aus einer ganz bestimmten Perspektive formuliert werden. In diesem Fall soll erklärt werden, warum Frauen "heute" die private Hausarbeit verrichten und so werden Frauen über die gesamte Menschheitsgeschichte hinweg „im Haus“ verortet. Es lassen sich jedoch keine direkten Linien von der Sammler*in zur Arbeiter*in oder Bürger*in nachweisen. Ganz im Gegenteil, hier zeigt sich, dass sowohl wissenschaftliche als auch populäre Bilder von der „Urgeschichte“ bis heute aus einem Blickwinkel entworfen werden, der die spezifischen Lebens- und Arbeitsformen der westlichen Gesellschaften seit dem 18. Jahrhundert voraussetzt. Entsprechend betont die Archäologin Brigitte Röder (2004, 2020), wie erstaunlich es ist, dass ein geschlechtsspezifisches Kulturmuster trotz erheblicher soziokultureller und ökologischer Veränderungen seit 400.000 Jahren konstant geblieben sein soll. Diese statische Sicht auf Geschichte wird durch ethnographische, 
soziologische und historische Studien über Arbeitsteilung längst in Frage gestellt (Röder 2004 , 2020; Palm 2012). Entsprechend gelangt Röder zu dem Schluss, dass die angeblich seit der Urgeschichte festgelegte Geschlechterordnung tatsächlich sehr jung und zudem eine bürgerliche Ordnung ist: „Die vermeintlich prähistorischen Kronzeugen dafür, dass der Mann eigentlich und ursprünglich die Rolle des Ernährers und die Frau die Rolle der Gattin, Hausfrau und Mutter habe, erweisen sich so überraschenderweise als ein bürgerliches Ehepaar“ (2020: 72). Diese historische Perspektive verdeutlicht, dass evolutionstheoretische Argumente zur Menschheitsgeschichte ohne einen Bezug zur Gesellschaftsgeschichte auskommen. Umgekehrt bekräftigt Röders Argumentation die Notwendigkeit einer gesellschaftswissenschaftlichen Fundierung und präzisen Kontextualisierung von vermeintlich ubiquitären Befunden, die Gesellschaft auf Umwelt reduzieren und das soziale Handeln von Menschen als Folge von biologischen und evolutionären Dispositionen erklären wollen.

\section{AUSBLICK}

In seiner diskursanalytischen Untersuchung der „Anlage-Umwelt-Debatten“ in den Erziehungswissenschaften plädiert Michael Lenz (2005) für eine weitere Annäherung zwischen geistes-, sozial- und naturwissenschaftlichem Wissen in der Sozialisationsforschung. Dieser Vorschlag ist unseres Erachtens zum jetzigen Zeitpunkt unrealistisch. Dies behaupten wir nicht wegen der gegenseitigen Vorwürfe, die letztlich nur von wenigen Stimmen vorgetragen werden. Unsere Skepsis resultiert vielmehr aus grundsätzlicheren Einwänden gegen ein bio-psycho-soziales Modell von Sozialisation, das zwar eine Interaktion von Anlagen und Umwelt beinhaltet, aber keinen Zugang zu Prozessen der Vergesellschaftung eröffnet. Denn die Stärke einer gesellschaftswissenschaftlichen Sozialisationstheorie liegt ja genau darin, dass sie komplexe Prozesse der Vergesellschaftung nicht auf einfache Kausalitätsmodelle mit berechenbaren Faktoren herunterbricht. Zugespitzt bedeutet das, dass die Sozialisation von Menschen nicht als "Disposition plus Umwelt" gedacht werden kann. Es geht, wie wir eingangs mit Berger und Luckmann betont haben, nicht um eine "biologische Natur des
Menschen", sondern um dessen komplexe, historisch und kulturell wandelbare Sozialität.

Deshalb gehen wir in Anlehnung an die erkenntniskritischen Debatten über Sozialisation und Geschlecht davon aus, dass Theorien, mit denen Wissenschaft dieses Verhältnis reflektieren will, selbst einer ständigen Reflexion unterzogen werden sollten: Welche Implikationen haben Sozialisationstheorien im Hinblick auf Konzeptionen von Gesellschaft, Subjekt, Identität und Körper? Solche Fragen werden in der sozial- und kulturwissenschaftlichen Geschlechterforschung seit den 1970er-Jahren kontrovers und intensiv verhandelt. Wie bereits angedeutet, haben sich daraus keine einheitlichen theoretischen Antworten auf die Frage, was Geschlechtersozialisation ausmacht, ergeben. Es gibt aber ausdifferenzierte theoretische und empirische Wissensbestände zum Verhältnis von Sozialisation und Geschlecht.

Die damit verbundenen theoretischen Suchbewegungen stehen im Gegensatz zu den vorgestellten naturwissenschaftlichen Zugängen. Für die analysierten Beispiele aus den interdisziplinären Neurowissenschaften zeigt sich, dass Gesellschaft auf Umwelt und soziale Faktoren beschränkt bleibt. Eine sozialwissenschaftliche Auseinandersetzung erfordert hingegen die Reflexion von Gesellschaft als komplexen Strukturund Ordnungszusammenhang (Gildemeister/Robert 2008; Becker-Schmidt 2017). Dieser Zusammenhang kann in der konkreten Sozialisationsforschung nur in Ausschnitten untersucht werden, bildet aber den heuristischen Kontext für diese Forschung.

In naturwissenschaftlichen Forschungssettings werden die Wahrnehmungen, Fähigkeiten, Leistungen, Verhaltensweisen und Organismen von Menschen analysiert. Belastbare Aussagen über das Verhältnis von Anlage und Umwelt sollen im Anschluss daran durch valide statistische Befunde abgesichert werden. Aus evolutionspsychologischer Sicht werden die überlebensnotwendigen Verhaltensimpulse des Menschen über Raum und Zeit hinweg universalisiert, selbst wenn sie in der Gegenwartsgesellschaft nicht mehr von Bedeutung sind. Bezogen auf Geschlecht gehen die vorgestellten Ansätze dabei fraglos von einer biologischen Zweigeschlecht- 
lichkeit und normativen Geschlechteridentitäten aus. Eine subjekttheoretische Fundierung von Sozialisation fokussiert hingegen die Qualität und den Verlauf von Aneignungs- und Verarbeitungsprozessen in konkreten gesellschaftlichen Zusammenhängen, sowohl intersubjektiv als auch intrasubjektiv (Becker-Schmidt/Knapp 1987; Rendtorff 2003; Bereswill 2014, 2018; Bereswill/Ehlert 2015; Quindeau 2018). Solche Prozesse lassen sich nicht messen und können auch nicht in experimentellen Forschungssettings untersucht werden. Sie bedürfen eines rekonstruktiven Theorieverständnisses (Dausien 2006). Das ist mit einem verstehenden Zugang zu den eigensinnigen und brüchigen Dimensionen von Geschlechtersozialisation verbunden (Maihofer 2002: 70).

Vor diesem Hintergrund erübrigt sich die Suche nach einheitlichen oder konsistenten Geschlechteridentitäten, wie sie in der Unterschiedsforschung vorausgesetzt werden. Geschlechtersozialisation wird vielmehr als die aktive und eigensinnige Auseinandersetzung des Subjekts mit den normativen Erwartungshorizonten einer gesellschaftlich dominanten Zweigeschlechtlichkeit verstanden.

\section{LITERATUR}

Althoff, Martina/Apel, Magdalena/Bereswill, Mechthild/Gruhlich, Julia/Riegraf, Birgit (2017): Feministische Methodologien und Methoden. Traditionen, Konzepte, Erörterungen. 2. Aufl. Wiesbaden: Springer VS. https://doi.org/10.1515/srsr-2019-0019

Baron-Cohen, Simon (2004): Vom ersten Tag an anders. Das weibliche und das männliche Gehirn. Düsseldorf/Zürich: Walter.

Becker-Schmidt, Regina (2017): Vergesellschaftung und Geschlecht. Soziologische Konzepte (1991). In: Regina Becker-Schmidt: Pendelbewegungen - Annäherungen an eine feministische Gesellschafts- und Subjekttheorie. Aufsätze aus den Jahren 1991-2015. Opladen/Berlin/Toronto: Barbara Budrich. S. 51-70. https://doi.org/10.2307/j.ctvddzggj.5

Becker-Schmidt, Regina/Knapp, Gudrun-Axeli (1987): Geschlechtertrennung - Geschlechterdifferenz. Suchbewegungen sozialen Lernens. Bonn: Neue Gesellschaft.
Bereswill, Mechthild (2008): Geschlecht. In: Baur, Nina; Korte, Hermann; Löw, Martina und Markus Schroer (Hrsg.): Handbuch Soziologie. Wiesbaden, S. 97-116. https://doi.org/10.1007/978-3531-91974-4_5

Bereswill, Mechthild (2014): Geschlecht als Konfliktkategorie. In: Behnke, Cornelia/Lengersdorf, Diana/Scholz, Sylka (Hrsg.): Wissen - Methode Geschlecht: Erfassen des fraglos Gegebenen. Wiesbaden: Springer VS, S.189-200. https://doi.org/10.1007/978-3-531-19654$\underline{1 \_13}$

Bereswill, Mechthild (2018): Geschlecht als Konfliktkategorie und als soziale Konstruktion. Überlegungen zu einer grundlegenden Spannung. In: Ahrbeck, Bernd/Dörr, Margret/Gstach, Johannes (Hrsg.): Der Genderdiskurs in der Psychoanalytischen Pädagogik. Eine notwendige Kontroverse. Jahrbuch für Psychoanalytische Pädagogik, Band 26. Gießen: Psychosozial-Verlag, S. 26-41. https://doi.org/10.30820/9783837974263-26

Bereswill, Mechthild/Ehlert, Gudrun (2015): Sozialisation im Kontext des Krisendiskurses über Jungen. In: Dausien, Bettina/Thon, Christine/Walgenbach, Katharina (Hrsg.): Geschlecht - Sozialisation - Transformation. Jahrbuch Frauen- und Geschlechterforschung in der Erziehungswissenschaft 11. Opladen/Berlin/Toronto: Barbara Budrich, S. 93-108. https://doi.org/10.3224/jfgfe.v11i1.05

Bereswill, Mechthild/ Gudrun Ehlert (2018): Geschlecht. In: Graßhoff, Gunther; Renker, Anna; Schröer, Wolfgang (Hrsg.): Soziale Arbeit. Eine elementare Einführung. Wiesbaden: Springer VS, S. 31-42. https://doi.org/10.1007/978-3658-15666-4_3

Berger, Peter L./Luckmann, Thomas (2004): Die gesellschaftliche Konstruktion der Wirklichkeit. Frankfurt am Main: Fischer Taschenbuch.

Bilden, Helga (1991): Geschlechtsspezifische Sozialisation. In: Hurrelmann, Klaus/Ulich, Dieter (Hrsg.): Handbuch der Sozialisationsforschung. Weinheim: Beltz, S. 281-303.

Bilden, Helga/Dausien, Bettina (Hrsg.) (2006): Sozialisation und Geschlecht. Theoretische und methodologische Aspekte. Opladen: Barbara Budrich. https://doi.org/10.2307/j.ctvm201fx.17 
Bischof-Köhler, Doris (2010): Evolutionäre Grundlagen geschlechtstypischen Verhaltens. In: Gisela Steins (Hrsg.): Handbuch Psychologie und Geschlechterforschung. Wiesbaden: VS, S. 153172.

Bischof-Köhler, Doris (2011): Von Natur aus anders. Die Psychologie der Geschlechtsunterschiede. 4., überarb. u. erw. Aufl. Stuttgart: Kohlhammer.

Bischof-Köhler, Doris (2012): Geschlechtstypisches Verhalten von Jungen aus evolutionstheoretischer und entwicklungspsychologischer Perspektive. In: Matzner, Michael/ Tischner, Wolfgang (Hrsg.): Handbuch Jungen-Pädagogik. Weinheim/Basel: Beltz, S. 50-65.

Bührmann, Andrea D./Diezinger, Angelika/MetzGöckel, Sigrid (2014): Arbeit - Sozialisation - Sexualität. Lehrbuch zur sozialwissenschaftlichen Frauen- und Geschlechterforschung. Wiesbaden: Springer VS. https://doi.org/10.1007/9783-531-19504-9

Butler, Judith (1991): Das Unbehagen der Geschlechter. Frankfurt am Main: Suhrkamp.

Cohen-Kettenis, Peggy T./van Goozen, Stephanie H. M./van Trotsenburg, Michael A. A. (2007): Das transsexuelle Gehirn. In: Lautenbacher, Stefan/Güntürkün, Onur/Hausmann, Markus (Hrsg.): Gehirn und Geschlecht. Neurowissenschaft des kleinen Unterschieds zwischen Frau und Mann. Heidelberg: Springer Medizin, S. 125-141. https://doi.org/10.1007/978-3-540-71628-0_7

Dausien, Bettina (2006): Geschlechterverhältnisse und ihre Subjekte. Zum Diskurs um Sozialisation und Geschlecht. In: Bilden, Helga/Dausien, Bettina (Hrsg.): Sozialisation und Geschlecht. Theoretische und methodologische Aspekte. Opladen: Barbara Budrich, S. 17-44. https://doi.org/10.2307/j.ctvbj7jtd.4

Ebeling, Smilla (2006): Wenn ich meine Hormone nehme, werde ich zum Tier. Zur Geschichte der "Geschlechtshormone“. In: Ebeling, Smilla/Schmitz, Sigrid (Hrsg.): Geschlechterforschung und Naturwissenschaften. Einführung in ein komplexes Wechselspiel. Wiesbaden: VS, S. 235-247. https://doi.org/10.1007/978-3531-90091-9_11

Ebeling, Smilla/Schmitz, Sigrid (Hrsg.) (2006): Geschlechterforschung
Naturwissenschaften. Einführung in ein komplexes Wechselspiel: Wiesbaden: VS.

Ehlert, Gudrun (2012): Gender in der Sozialen Arbeit. Konzepte, Perspektiven, Basiswissen. Schwalbach am Taunus: Wochenschau.

Gildemeister, Regine/Hericks, Katja (2012): Geschlechtersoziologie. Theoretische Zugänge zu einer vertrackten Kategorie des Sozialen. München:

Oldenbourg.

https://doi.org/10.1524/9783486717570

Gildemeister, Regine/Robert, Günther (2008): Geschlechterdifferenzierungen in lebenszeitlicher Perspektive. Interaktion-Institution-Biografie. Wiesbaden: VS.

Gildemeister, Regine/Wetterer, Angelika (1992): Wie Geschlechter gemacht werden. Die soziale Konstruktion der Zweigeschlechtlichkeit und ihre Reifizierung in der Frauenforschung. In: Knapp, Gudrun-Axeli/Wetterer, Angelika (Hrsg.): TraditionenBrüche. Entwicklungen feministischer Theorie. Freiburg im Breisgau: Kore, S. 201-254.

Haider, Hilde/Malberg, Ewelina D. (2010): Sollten geschlechtsspezifische Unterschiede in der Allgemeinen Psychologie berücksichtigt werden? In: Gisela Steins (Hrsg.): Handbuch Psychologie und Geschlechterforschung. Wiesbaden: VS, S. 105-130. https://doi.org/10.1007/978-3-53192180-8_7

Hagemann-White, Carol (1984): Sozialisation: Weiblich - männlich? Opladen: Leske und Budrich. $\quad$ https://doi.org/10.1007/978-3-32297160-9

Hausmann, Markus (2007): Kognitive Geschlechtsunterschiede. In: Lautenbacher, Stefan/Güntürkün, Onur/Hausmann, Markus (Hrsg.): Gehirn und Geschlecht. Neurowissenschaft des kleinen Unterschieds zwischen Frau und Mann. Heidelberg: Springer Medizin, S. 106-123. https://doi.org/10.1007/978-3-531-92180-8_5

Hirnstein, Marco/Hausmann, Markus (2010): Kognitive Geschlechtsunterschiede. In: Gisela Steins (Hrsg.): Handbuch Psychologie und Geschlechterforschung. Wiesbaden: VS, S. 78-85.

Holsboer, Florian (2007): Geleitwort. In: Lautenbacher, Stefan/Güntürkün, Onur/Hausmann, Markus (Hrsg.) (2007): Gehirn und Geschlecht. Neurowissenschaft des kleinen Unterschieds zwischen Frau und Mann. Heidelberg: Springer 
Medizin, S. V. https://doi.org/10.1007/978-3$\underline{540-71628-0}$

Hurrelmann, Klaus (1983): Das Modell des produktiv realitätsverarbeitenden Subjekts in der Sozialisationsforschung. In: Zeitschrift für Sozialisationsforschung und Erziehungssoziologie 3 (1), S. 91-103. https://doi.org/10.1007/978-3$\underline{531-92715-2 \_4}$

Hustvedt, Siri (2018): Die Illusion der Gewissheit. Reinbek bei Hamburg: Rowohlt.

Hyde, Janet Shibley (2005): The Gender Similarities Hypothesis In: American Psychologist 60 (6), S. 581-509.

Jordan, Kirsten (2010): Gehirn zwischen Sex und Gender - Frauen und Männer aus neurowissenschaftlicher Perspektive. In: Steins, Gisela (Hrsg.): Handbuch Psychologie und Geschlechterforschung. Wiesbaden: VS, S. 87-104.

Kasten, Hartmut (2012): Entwicklungspsychologische Aspekte der Erziehung und Bildung von Jungen. In: Matzner, Michael/Tischner, Wolfgang (Hrsg.): Handbuch Jungen-Pädagogik. Weinheim/Basel: Beltz, S. 66-79.

Knapp, Gudrun-Axeli (2014): Arbeiten am Unterschied. Eingriffe feministischer Theorie. Innsbruck/Wien/Bozen: StudienVerlag. https://doi.org/10.1007/s11614-014-0141-7

Kortendiek, Beate/Riegraf, Birgit/Sabisch, Katja (Hrsg.) (2019): Handbuch interdisziplinäre Geschlechterforschung. Wiesbaden: Springer VS. https://doi.org/10.1515/fs-2019-0041

Krebs, Uwe (2012): Interdisziplinarität bei der wissenschaftlichen Behandlung der Geschlechterthematik. In: Matzner, Michael/Tischner, Wolfgang (Hrsg.): Handbuch Jungen-Pädagogik. Weinheim/Basel: Beltz, S. 18-34.

Lautenbacher, Stefan/Güntürkün, Onur/Hausmann, Markus (Hrsg.) (2007): Gehirn und Geschlecht. Neurowissenschaft des kleinen Unterschieds zwischen Frau und Mann. Heidelberg: Springer Medizin. https://doi.org/10.1007/978-3-540-71628-0

Lenz, Karl (2011): Sozialisation. In: Ehlert, Gudrun/Funk, Heide/Stecklina, Gerd (Hrsg.) (2011): Wörterbuch Soziale Arbeit und Geschlecht. Weinheim/München: Juventa, S. 385-387.
Lenz, Michael (1999): Geschlechtersozialisation aus biologischer Sicht. Anlage und Erziehung. Stuttgart: ibidem.

Lenz, Michael (2005): Die Diskussion über Anlage und Umwelt in der bundesdeutschen Erziehungswissenschaft aus der diskursanalytischen Perspektive. In: ZSE: Zeitschrift für Soziologie der Erziehung und Sozialisation 25 (4), S. 340-361.

Lutchmaya, Svetlana/Baron-Cohen, Simon (2002): Human Sex Differences in Social and Non-Social Looking Preferences, at 12 Months of Age. In: Infant Behavior \& Development 25, S. 319-325. https://doi.org/10.1016/s01636383(02)00095-4

Maihofer, Andrea (2002): Geschlecht und Sozialisation. Eine Problemskizze. In: Erwägen Wissen Ethik (EWE) 13 (1), S. 13-26.

Matzner, Michael/Tischner, Wolfgang (Hrsg.) (2008): Handbuch Jungen-Pädagogik. Weinheim/Basel: Beltz.

Matzner, Michael/Tischner, Wolfgang (2008): Einleitung. In: Matzner, Michael/Tischner Wolfgang (Hrsg.): Handbuch Jungen-Pädagogik. Weinheim/Basel: Beltz, S. 9-15.

Matzner, Michael/Tischner, Wolfgang (Hrsg.) (2012): Handbuch Jungen-Pädagogik. 2. erw. Aufl. Weinheim/Basel: Beltz.

Metz-Göckel, Sigrid (2000): Sozialisation der Geschlechter: Von der Geschlechterdifferenz zur Dekonstruktion der Geschlechterdualität. In: Bührmann, Andrea D./Diezinger, Angelika/MetzGöckel, Sigrid: Arbeit, Sozialisation, Sexualität. Zentrale Felder der Frauen- und Geschlechterforschung. Opladen: Leske und Budrich, S. 103192. https://doi.org/10.1007/978-3-53119504-9_2

Palm, Kerstin (2008): Biologie: Geschlechterforschung zwischen Reflektion und Intervention. In: Becker, Ruth/Kortendiek, Beate (Hrsg.): Handbuch Frauen- und Geschlechterforschung. Theorie, Methoden, Empirie. 2., erw. Aufl. Wiesbaden: VS, S. 843-851. https://doi.org/10.1007/978-3531-91972-0_101

Palm, Kerstin (2012): Grundlagen und Visionen einer genderreflexiven Biologiedidaktik. In: Kampshoff, Marita/Wiepcke, Claudia (Hrsg.): Handbuch Geschlechterforschung und 
Fachdidaktik. Wiesbaden: VS, S. 69-82. https://doi.org/10.1007/978-3-531-18984-0_6

Palm, Kerstin (2020): Gibt es geschlechtsspezifische kognitive Fähigkeiten? In: Rendtorff, Barbara/Mahs, Claudia/Warmuth, Anne-Dorothee (Hrsg.): Geschlechterverwirrungen. Was wir wissen, was wir glauben und was nicht stimmt. Frankfurt am Main/New York: Campus. S. 16067.

Pauen, Sabina (Hrsg.) (2016): Entwicklungspsychologie im Kindes- und Jugendalter. Berlin/Heidelberg: Springer.

Quindeau, Ilka (2018): Von normativen Identitätsvorstellungen zur Ambiguitätstoleranz. In: Ahrbeck, Bernd/Dörr, Margret/Gstach, Johannes (Hrsg.): Der Genderdiskurs in der Psychoanalytischen Pädagogik. Eine notwendige Kontroverse. Jahrbuch für Psychoanalytische Pädagogik, Band 26. Gießen: Psychosozial-Verlag, S. 12-25. https://doi.org/10.30820/9783837974263-12

Rendtorff, Barbara (2003): Kindheit, Jugend und Geschlecht. Einführung in die Psychologie der Geschlechter. Weinheim/Basel/Berlin: Beltz Taschenbuch.

Rieker, Peter (2013): Sozialisation und Geschlecht - Theoretische Herausforderung und Perspektiven der empirischen Sozialforschung. In: Mechthild Bereswill, Mechthild/Liebsch, Katharina (Hrsg.) (2013): Geschlecht (re)konstruieren. Zur methodologischen und methodischen Produktivität der Frauen- und Geschlechterforschung. Münster: Westfälisches Dampfboot, S. 42-60.

Röder, Brigitte (2004): Frauen, Kinder und andere Minderheiten. Alter und Geschlecht auf Lebensbildern zur Urgeschichte. In: Ethnographisch-Archäologische Zeitschrift 45 (2-3), S. 507-520.

Röder, Brigitte (2020): Beutejäger und Nesthüterin - trügerische Orientierung an einem steinzeitlichen Traumpaar. In: Rendtorff, Barbara/Mahs, Claudia/Warmuth, Anne-Dorothee (Hrsg.): Geschlechterverwirrungen. Was wir wissen, was wir glauben und was nicht stimmt. Frankfurt am Main/New York: Campus, S. 69-76.

Scheu, Ursula (1977): Wir werden nicht als Mädchen geboren - wir werden dazu gemacht. Zur frühkindlichen Erziehung in unserer Gesellschaft. Frankfurt am Main: Fischer Taschenbuch.

Schmitz, Sigrid/Höppner, Grit (2014): Feminist Neuroscience: a Critical Review of Contemporary Brain Research. In: Frontiers in Human Neuroscience

8:546

https://doi.org/10.3389/fnhum.2014.00546

Siegler, Robert/Eisenberg, Nancy/DeLoache, Judy/Saffran, Jenny (2016): Die Entwicklung der Geschlechter. In: Pauen, Sabina (Hrsg.): Entwicklungspsychologie im Kindes- und Jugendalter. Berlin/Heidelberg: Springer, S. 575-617. https://doi.org/10.1007/978-3-662-470282_15

Steins, Gisela (Hrsg.) (2010): Handbuch Psychologie und Geschlechterforschung. Wiesbaden: VS.

Strüber, Daniel (2012): Geschlechtsunterschiede im Verhalten und ihre hirnbiologischen Grundlagen. In: Matzner, Michael/Tischner, Wolfgang (Hrsg.): Handbuch Jungen-Pädagogik. Weinheim/, Basel: Beltz, S. 35-49.

Tervooren, Anja (2007): Einüben von Geschlecht und Begehren. Plädoyer für eine rekonstruktive Sozialisationstheorie. In: Feministische Studien, 25 (1), S. 40-54. https://doi.org/10.1515/fs2007-0105

von Uexküll, Jakob (1956): Streifzüge durch die Umwelten von Tieren und Menschen. Ein Bilderbuch unsichtbarer Welten. Reinbek bei Hamburg: Rohwolt. https://doi.org/10.1007/978-3642-98976-6_1

Trepte, Sabine/ Reinecke, Leonard (2010): Medienpsychologie. Gender und Games - Medienpsychologische Gender-Forschung am Beispiel Video- und Computerspiele. In: Steins, Gisela (Hrsg.): Handbuch Psychologie und Geschlechterforschung. Wiesbaden: VS-Verlag. S. 229248. $\quad$ https://doi.org/10.1007/978-3-53192180-8_13

Williams, Christina L./Pleil, Kristen E. (2008): Toy Story: Why Do Monkey and Human Males Prefer Trucks? Comment on "Sex Differences in Rhesus Monkey Toy Preferences Parallel Those of Children" by Hassett, Siebert, and Wallen. In: Hormones and Behavior 54 (3), S. 355-358. https://doi.org/10.1016/j.yhbeh.2008.05.003 
Zu den Autorinnen

Mechthild Bereswill ist Professorin der Soziologie sozialer Differenzierung und Soziokultur an der Universität Kassel, Fachbereich Humanwissenschaften (01). Ihre Arbeitsschwerpunkte in Forschung und Lehre sind Soziologie und Sozialpsychologie der Geschlechterverhältnisse; soziale Probleme und soziale Kontrolle sowie qualitative Methodologien.

Gudrun Ehlert ist Professorin der Sozialarbeitswissenschaft an der Hochschule Mittweida (University of Applied Sciences), Fakultät Soziale Arbeit. Ihre Arbeitsschwerpunkte in Forschung und Lehre sind Geschlechterverhältnisse in der Sozialen Arbeit, Profession und Geschlecht, Professionalisierung Sozialer Arbeit sowie Soziale Ungleichheit.

\section{Kontakt}

Prof. Dr. phil. habil. Mechthild Bereswill

Universität Kassel

Gottschalkstraße 10-12

34127 Kassel

Tel.: +49 5618042976

E-Mail: bereswill@uni-kassel.de

URL: https://www.uni-kassel.de/fb01/institute/sozialwesen/fachgebiete/soziologie-sozialer-differenzierung-und-soziokultur/prof-dr-mechthild-bereswill.html

Prof. Dr. phil. Gudrun Ehlert

Hochschule Mittweida

Fakultät Soziale Arbeit

Zentrum Medien und Soziale Arbeit (ZMS)

Bahnhofstr. 15

09648 Mittweida

Tel.: +493727581703

E-Mail: ehlert@hs-mittweida.de

URL: https://www.sw.hs-mittweida.de/professuren/prof-dr-phil-gudrun-ehlert.html 